\title{
Lower Bounds in Real Algebraic Geometry and Orientability of Real Toric Varieties
}

\author{
Evgenia Soprunova - Frank Sottile
}

Received: 10 November 2012 / Revised: 9 March 2013 / Accepted: 19 March 2013 /

Published online: 3 April 2013

(C) Springer Science+Business Media New York 2013

\begin{abstract}
The real solutions to a system of sparse polynomial equations may be realized as a fiber of a projection map from a toric variety. When the toric variety is orientable, the degree of this map is a lower bound for the number of real solutions to the system of equations. We strengthen previous work by characterizing when the toric variety is orientable. This is based on work of Nakayama and Nishimura, who characterized the orientability of smooth real toric varieties.
\end{abstract}

Keywords Real toric variety $\cdot$ Polynomial system $\cdot$ Order polytope

Mathematics Subject Classification (1991) $14 \mathrm{M} 25 \cdot 57 \mathrm{~B} 20 \cdot 57 \mathrm{~S} 10 \cdot 14 \mathrm{P} 99$

\section{Introduction}

A ubiquitous phenomenon in enumerative real algebraic geometry is that many geometric problems possess a non-trivial lower bound on their number of real solutions. For example, at least 3 of the 27 lines on a real cubic surface are real as are at least 8 of the 12 rational cubics interpolating 8 real points in the plane [4, Prop. 4.7.3], but there are many, many other examples $[1,6,7,9,16,17,19,20,23]$. This phenomenon has the potential for significant impact on the applications of mathematics as a nontrivial lower

\footnotetext{
E. Soprunova

Department of Mathematics, Kent State University, Summit Street, Kent, OH 44242, USA

e-mail: soprunova@math.kent.edu

URL: http://www.math.kent.edu/ soprunova/

F. Sottile $(\varangle)$

Department of Mathematics, Texas A\&M University, College Station, TX 77843, USA

e-mail: sottile@math.tamu.edu

URL: http://www.math.tamu.edu/ sottile
} 
bound is an existence proof of real solutions. For this potential to be realized, methods need to be developed to predict when a system of polynomial equations or a geometric problem has a lower bound on its number of real solutions and to compute this bound.

We developed a theory of lower bounds on the number of real solutions to systems of sparse polynomials [20]. There, a system of polynomial equations was formulated as a fiber of a projection map from a toric subvariety of a sphere. When the toric variety is orientable, the absolute value of the degree of this projection map is a lower bound on the number of real solutions. Besides giving a condition implying this orientability, a method (foldable triangulations of the Newton polytope) was developed to compute the degree of certain maps, and a class of examples of polynomial systems (Wronski polynomial systems from posets) was presented to which this theory applied.

Further work $[12,13]$ on foldable triangulations has advanced our understanding of the bound they give. Others $[1,7,11,16,17]$ have developed additional methods for proving lower bounds in real algebraic geometry and experimentation $[10,19,20]$ has revealed many more likely examples of lower bounds.

We characterize which sparse polynomial systems possess a lower bound in the context of [20], by extending work of Nakayama and Nishimura [15], who characterized the orientability of small covers, which are topological versions of smooth real projective toric varieties. We characterize the orientability of the smooth points of any real toric variety, as well as toric subvarieties of a sphere, solving an important open problem from [20].

We review the construction of real toric varieties and spherical toric varieties in Sect. 2, where we formulate our results on orientability. Section 3 contains the mildly technical proof of these results. In Sect. 4 we use this characterization of orientability to strengthen results from [20] on the theory of lower bounds for the number of real solutions to systems of sparse polynomials.

\section{Constructions of Real Toric Varieties}

Real toric varieties appear in many applications of mathematics $[2,14,18]$ and are interesting objects in their own right [5]. Davis and Januszkiewicz [3] introduced the notion of a small cover of a simple convex polytope as a generalization of smooth projective real toric varieties. We describe real toric varieties and small covers in terms of the gluing of explicit cell complexes and give a mild extension of Davis and Januszkiewicz's notion of a small cover (which are manifolds) to not necessarily smooth spaces. A projective toric variety may be lifted to the sphere over real projective space, and we also describe these spherical toric varieties in terms of the gluing of explicit cell complexes.

Nakayama and Nishimura [15] used this presentation of small covers to characterize their orientability, and similar arguments characterize the orientability of the smooth points of the above spaces.

Real toric varieties, singular small covers, and toric subvarieties of the sphere are obtained by gluing the real torus $\mathbb{T}^{n}:=\left(\mathbb{R}^{\times}\right)^{n}$ or $\{ \pm 1\} \times \mathbb{T}^{n}=\mathbb{T}^{n+1} / \mathbb{R}_{\geq}$along copies 
of $\mathbb{T}^{n-1}$, one copy for each vector in a set of integer vectors. There are further gluings in higher codimension, which presents these spaces as explicit cell complexes. They are smooth at the points of their dense torus $\mathbb{T}^{n}$ (or at $\{ \pm 1\} \times \mathbb{T}^{n}$ ) and the attached tori $\mathbb{T}^{n-1}$, and so their orientability is determined by the gluing along the tori $\mathbb{T}^{n-1}$.

Complex toric varieties are normal varieties over $\mathbb{C}$ equipped with an action of an algebraic torus $\left(\mathbb{C}^{\times}\right)^{n}$ having a dense orbit. They are classified by rational fans $\Sigma$ in $\mathbb{R}^{n}$, which encode their construction as a union of affine toric varieties $U_{\sigma}$, one for each cone $\sigma \in \Sigma$. A toric variety is a union of disjoint torus orbits $\mathcal{O}_{\sigma}$, one for each cone $\sigma \in \Sigma$, with $\operatorname{dim} \mathcal{O}_{\sigma}=n-\operatorname{dim}(\sigma)$. The dense orbit $\mathcal{O}_{0}$ coincides with the smallest affine patch $U_{0}$, and both are associated to the origin 0 in the fan. See [8] for a complete description.

A toric variety has a canonical set $Y$ of real points obtained from the real points of the orbits $\mathcal{O}_{\sigma}$ of the construction $\left[8\right.$, Ch. 4]. The dense orbit $\mathcal{O}_{0}(\mathbb{R}) \simeq \mathbb{T}^{n}$ is isomorphic to $\left(\mathbb{R}^{\times}\right)^{n}=\left(\mathbb{R}_{>0}\right)^{n} \times\{ \pm 1\}^{n}$, which has $2^{n}$ components, each a topological $n$-ball. The subgroup $\{ \pm 1\}^{n} \subset \mathbb{T}^{n}$ acts on $Y$, permuting the components of $\mathcal{O}_{0}(\mathbb{R})$. The orbit space of $Y$ under the group $\{ \pm 1\}^{n}$ is isomorphic to the closure $Y_{\geq}$of any component of $\mathcal{O}_{0}(\mathbb{R})$ in the usual topology (not Zariski!) on $Y$. Each orbit $\mathcal{O}_{\sigma}(\mathbb{R})$ has a unique component contained in $Y_{\geq}$. We call this component $F_{\sigma}$ a face of $Y_{\geq}$, which is isomorphic to $\left(\mathbb{R}_{>0}\right)^{n-\operatorname{dim}(\sigma)}$. This endows $Y_{\geq}$with the structure of a cell complex that is dual to the fan $\Sigma$. That is, the intersection $\overline{F_{\sigma}} \cap \overline{F_{\tau}}$ of the closures of two faces is nonempty only if $\sigma$ and $\tau$ lie in some cone of $\Sigma$, in which case it is the closure $\overline{F_{\rho}}$ where $\rho$ is the minimal such cone.

The integer points in a cone $\sigma$ of $\Sigma$ form a subsemigroup of $\mathbb{Z}^{n}$ whose image in $(\mathbb{Z} / 2 \mathbb{Z})^{n}=\{ \pm 1\}^{n}$ is a subgroup $\bar{\sigma}$ of $\{ \pm 1\}^{n}$. This subgroup $\bar{\sigma}$ is the isotropy subgroup of the face $F_{\sigma}$ of $Y_{\geq}$. We will write $(-1)^{v}=\left((-1)^{v_{1}}, \ldots,(-1)^{v_{n}}\right)$ for the image of $v \in \mathbb{Z}^{n}$ in $(\mathbb{Z} / 2 \mathbb{Z})^{n}=\{ \pm 1\}^{n}$. This gives the following description of $Y$ as a quotient space of $Y_{\geq} \times\{ \pm 1\}^{n}$.

Proposition 2.1 The real toric variety $Y$ is obtained as the quotient of the cell complex $Y_{\geq} \times\{ \pm 1\}^{n}$ by the equivalence relation where

$$
(p, \xi) \sim(q, \eta) \Longleftrightarrow p=q \text { and } \xi \bar{\sigma}=\eta \bar{\sigma} \text {, where } p \text { lies in the face } F_{\sigma} .
$$

A facet of $Y_{\geq}$is a face $F_{\sigma}$ corresponding to a one-dimensional cone $\sigma$. The real toric variety $Y$ is smooth at points corresponding to facets, but may not be smooth along lower-dimensional faces. If $Y_{\geq}^{\circ}$ is the union of the dense face $F_{0}$ and its facets, then

$$
Y^{\circ}:=\left(Y_{\geq}^{\circ} \times\{ \pm 1\}^{n}\right) / \sim
$$

consists of smooth points of $Y$.

We generalize this construction. Let $P$ be a finite ranked poset with minimal element 0 and rank at most $n$ where two elements $\sigma, \tau \in P$ have at most one minimal upper bound in $P$. The cones $\sigma$ in a rational fan in $\mathbb{R}^{n}$ form such a poset. Suppose further that we have a collection $\mathcal{S}:=\{\bar{\sigma} \mid \sigma \in P\}$ of subgroups of $\{ \pm 1\}^{n}$ where $\bar{\sigma} \simeq\{ \pm 1\}^{\operatorname{rank}(\sigma)}$, and if $\sigma \subset \tau$, then $\bar{\sigma} \subset \bar{\tau}$. Finally, suppose that we have a cell complex $\Delta$ with cells (called faces) indexed by elements of $P$, 


$$
\Delta=\coprod_{\sigma \in P} F_{\sigma},
$$

where each face $F_{\sigma}$ is a cell of dimension $n-\operatorname{rank}(\sigma)$, which we identify with the interior of the closed unit ball in $\mathbb{R}^{n-\operatorname{rank}(\sigma)}$. We further suppose that:

- $\Delta$ is a subset of the closed ball $\overline{F_{0}}$ in $\mathbb{R}^{n}$,

- the closure of a face $F_{\sigma}$ in $\mathbb{R}^{n}$ is homeomorphic to the closed ball of dimension $n-\operatorname{rank}(\sigma)$, and

- given $\sigma, \tau \in P$, the closures of the faces $F_{\sigma}$ and $F_{\tau}$ either do not meet (if $\sigma$ and $\tau$ have no upper bound in $P$ ), or their intersection is the closure of the face $F_{\rho}$, where $\rho$ is the least upper bound of $\sigma$ and $\tau$ in $P$.

Definition 2.2 Given a ranked poset $P$, system $\mathcal{S}$ of subgroups of $\{ \pm 1\}^{n}$, and a cell complex $\Delta$ as above, the small cover $Y(\Delta, \mathcal{S})$ of $\Delta$ is the quotient

$$
\left(\Delta \times\{ \pm 1\}^{n}\right) / \sim
$$

where $(p, \xi) \sim(q, \eta)$ if and only if $p=q$ and $\xi \bar{\sigma}=\eta \bar{\sigma}$, where $p$ lies in the face $F_{\sigma}$.

Observe that $Y(\Delta, \mathcal{S})$ is equipped with a natural action of $\{ \pm 1\}^{n}$ whose orbit space is $\Delta$, where the orbit of a face $F_{\sigma}$ is identified with $F_{\sigma} \times\{ \pm 1\}^{n} / \bar{\sigma} \simeq \mathbb{T}^{n-\operatorname{rank}(\sigma)}$. In particular, it is a $\{ \pm 1\}^{n}$-equivariant compactification of $\mathbb{T}^{n}$.

A real toric variety $Y$ associated to a fan $\Sigma$ is a small cover where $P$ is the set of cones in the fan, $\Delta=Y_{\geq}$, and $\mathcal{S}=\{\bar{\sigma} \mid \sigma \in \Sigma\}$.

The points of $Y(\Delta, \mathcal{S})$ corresponding to the big cell $F_{0}$ and to facets $F_{\sigma}$ are points where $Y(\Delta, \mathcal{S})$ is a topological manifold. Write $\Delta^{\circ}$ for the union of the big cell and the facets, and $Y^{\circ}(\Delta, \mathcal{S})=\left(\Delta^{\circ} \times\{ \pm 1\}^{n}\right) / \sim$ for this subset of the smooth points of $Y(\Delta, \mathcal{S})$.

Let $\Delta \subset \mathbb{R}^{n}$ be a $n$-dimensional polytope with integer vertices and normal fan $\Sigma$. Then the real toric variety $Y_{\Sigma}$ associated to $\Sigma$ has a projective embedding given by $\Delta$. We may assume that the integer points $\Delta \cap \mathbb{Z}^{n}$ generate $\mathbb{Z}^{n}$. Let $\mathbb{P}^{\Delta}$ be the real projective space with coordinates indexed by $\Delta \cap \mathbb{Z}^{n}$ and $y^{\alpha}:=y_{1}^{\alpha_{1}} \cdots y_{n}^{\alpha_{n}}$ the monomial with exponent $\alpha$. Then we have an injection

$$
\varphi_{\Delta}: \mathbb{T}^{n} \ni y \longmapsto\left[y^{\alpha} \mid \alpha \in \Delta \cap \mathbb{Z}^{n}\right]
$$

where $[\cdots]$ denotes homogeneous coordinates for $\mathbb{P}^{\Delta}$. The closure $Y_{\Delta}$ of the image of this map is isomorphic to the real toric variety $Y_{\Sigma}$, and the cell complex $Y_{\geq}^{\circ}$ is identified with the polytope $\Delta$.

The unit sphere $\mathbb{S}^{\Delta} \subset \mathbb{R}^{\Delta}$ has a two-to-one map to $\mathbb{P}^{\Delta}$, and we define $Y_{\Delta}^{+}$to be the pullback of $Y_{\Delta}$ along this map. The sphere $\mathbb{S}^{\Delta}$ has homogeneous coordinates $\left(x_{\alpha} \mid\right.$ $\alpha \in \Delta \cap \mathbb{Z}^{n}$ ), where we identify points with a positive constant of proportionality. The group $\{ \pm 1\}^{n+1}$ acts on $\mathbb{S}^{\Delta}$ with the last coordinate acting through global multiplication by \pm 1 and the remaining coordinates $\{ \pm 1\}^{n}$ through the map $\varphi_{\Delta}$ (2.1),

$$
\left(g, g_{n+1}\right) .\left(x_{\alpha} \mid \alpha \in \Delta \cap \mathbb{Z}^{n}\right)=\left(g_{n+1} g^{\alpha} x_{\alpha} \mid \alpha \in \Delta \cap \mathbb{Z}^{n}\right) .
$$


The faces of $Y_{\Delta}^{+}$are its intersections with coordinate subspaces $\mathbb{S}^{F}$ of $\mathbb{S}^{\Delta}$ corresponding to faces $F$ of $\Delta$,

$$
\mathbb{S}^{F}:=\left\{\left(x_{\alpha} \mid \alpha \in \Delta \cap \mathbb{Z}^{n}\right) \mid x_{\alpha}=0 \text { if } \alpha \notin F \cap \mathbb{Z}^{n}\right\} .
$$

The isotropy subgroup of $\mathbb{S}^{F}$ is

$$
\left\{\left(g, g_{n+1}\right) \mid g_{n+1} g^{\alpha}=1 \text { for } \alpha \in F \cap \mathbb{Z}^{n}\right\} .
$$

Vectors $b$ in the normal cone $\sigma_{F}$ to a face $F$ of $\Delta$ have constant dot product with elements of $F$-define $b \cdot F$ to be this constant. Then the subgroup

$$
\bar{\sigma}_{F}+:=\left\{(-1)^{(b, b \cdot F)} \mid b \in \sigma_{F}\right\} \subset\{ \pm 1\}^{n+1}
$$

is the isotropy group of $\mathbb{S}^{F}$, and therefore of the corresponding face of $Y_{\Delta}^{+}$.

Proposition 2.3 The spherical toric variety $Y_{\Delta}^{+}$is obtained as the quotient of the cell complex $\Delta \times\{ \pm 1\}^{n+1}$ by the equivalence relation

$$
(p, \xi) \sim(q, \eta) \Longleftrightarrow p=q \text { and } \xi \bar{\sigma}_{F}{ }^{+}=\eta \bar{\sigma}_{F}{ }^{+} \text {, where } p \text { lies in the face } F \text {. }
$$

\section{Characterization of Orientability}

We follow Nakayama and Nishimura [15] to characterize the orientability of a general small cover and of spherical toric varieties, and determine their numbers of components.

Theorem 3.1 Let $Y(\Delta, S)$ be a small cover of dimension $n$.

(1) $Y^{\circ}(\Delta, S)$ is orientable if and only if there exists a basis of $\{ \pm 1\}^{n}$ such that for every $\sigma \in P$ of rank 1 , the generator of $\bar{\sigma} \simeq\{ \pm 1\}$ is a product of an odd number of basis vectors.

(2) The components of $Y^{\circ}(\Delta, S)$ are naturally indexed by $\{ \pm 1\}^{n} /\langle\bar{\sigma} \mid \operatorname{rank}(\sigma)=1\rangle$.

Thus $Y^{\circ}(\Delta, S)$ has $2^{n-k}$ connected components, where $2^{k}=|\langle\bar{\sigma} \mid \operatorname{rank}(\sigma)=1\rangle|$.

Proof For each $\sigma \in P$ with rank 1 , let $g_{\sigma}$ be the generator of $\bar{\sigma} \simeq \mathbb{Z} / 2 \mathbb{Z}$. Then $Y^{\circ}:=Y^{\circ}(\Delta, S)$ is obtained by gluing $(\Delta, \xi)$ and $(\Delta, \eta)$ along $F_{\sigma}$ whenever $\xi=\eta g_{\sigma}$ for some $\sigma \in P$ of rank 1 , so the connected components of $Y^{\circ}$ correspond to the orbits of $Y^{\circ}$ under the action of $\langle\bar{\sigma} \mid \operatorname{rank}(\sigma)=1\rangle$.

The space $Y^{\circ}$ is orientable if and only if $H_{n}\left(Y^{\circ}, \mathbb{Z}\right) \neq\{0\}$. This group is the kernel ker $\partial$ of the differential in the cellular chain complex of the cell complex $Y^{\circ}$,

$$
C_{n} \stackrel{\partial}{\longrightarrow} C_{n-1}
$$

Here $C_{n}$ is the free abelian group generated by

$$
\{\Delta\} \times\{ \pm 1\}^{n}=\left\{(\Delta, \xi) \mid \xi \in\{ \pm 1\}^{n}\right\}
$$


and $C_{n-1}$ is the free abelian group generated by

$$
\left\{\left[F_{\sigma}, \xi\right] \mid \sigma \in P, \operatorname{rank}(\sigma)=1, \xi \in\{ \pm 1\}^{n}\right\} / \sim
$$

where $\left[F_{\sigma}, \xi\right] \sim\left[F_{\sigma}, \xi g_{\sigma}\right]$. Orient each facet $F_{\sigma}$ so that

$$
\partial(\Delta)=\sum_{\operatorname{rank}(\sigma)=1} F_{\sigma}
$$

Consider an $n$-cycle

$$
X=\sum_{\xi \in\{ \pm 1\}^{n}} n_{\xi} \cdot(\Delta, \xi) \in C_{n}
$$

on $Y^{\circ}$, where $n_{\xi} \in \mathbb{Z}$. Then

$$
\partial(X)=\sum_{\xi \in\{ \pm 1\}^{n}} n_{\xi} \sum_{\operatorname{rank}(\sigma)=1}\left[F_{\sigma}, \xi\right]=\sum_{\operatorname{rank}(\sigma)=1} \sum_{\xi \in\{ \pm 1\}^{n} /\left\langle g_{\sigma}\right\rangle}\left(n_{\xi}+n_{\xi g_{\sigma}}\right)\left[F_{\sigma}, \xi\right]
$$

Hence an $n$-cycle $X$ lies in ker $\partial$ if and only if $n_{\xi}=-n_{\xi g_{\sigma}}$ for all $\xi$ in $\{ \pm 1\}^{n}$ and $\sigma$ of rank 1. Equivalently, $n_{\xi}=(-1)^{k} n_{\xi g_{\sigma_{1}} \cdots g_{\sigma_{k}}}$ for all $\xi \in\{ \pm 1\}^{n}$ and $\sigma_{i}$ of rank 1 .

We show that ker $\partial$ is non-trivial if and only if there exists a basis $e_{1}, \ldots, e_{n}$ of $\{ \pm 1\}^{n}$ such $g_{\sigma}$ is a product of an odd number of basis vectors, for each element $\sigma \in P$ of rank one. Let $\mathbb{O}$ be the set of generators $g_{\sigma}$ of $\bar{\sigma}$ for rank one elements $\sigma \in P$.

Suppose that there exists a basis $e_{1}, \ldots, e_{n}$ of $\{ \pm 1\}^{n}$ such that each $g_{\sigma} \in \mathbb{O}$ is a product of an odd number of basis vectors. For $\xi \in\{ \pm 1\}^{n}$ define $n_{\xi}$ to be 1 if $\xi$ is a product of an even number of the $e_{i}$ and -1 if it is a product of an odd number of the $e_{i}$. Then $n_{\xi}=-n_{\xi g_{\sigma}}$ for all $\xi$ and $\sigma$, so ker $\partial$ is non-trivial and hence $Y^{\circ}$ is orientable. Since the number of connected components is $2^{n-k}$, the kernel is isomorphic to $\mathbb{Z}^{2^{n-k}}$.

If there is no such basis of $\{ \pm 1\}^{n}$, then there is some $g_{\sigma} \in \mathbb{O}$ which is a product of an even number of other elements in $\mathbb{O}$, for otherwise we can reduce $\mathbb{O}$ to a linearly independent set and then extend it to a basis of $\{ \pm 1\}^{n}$. We get $g_{\sigma}=g_{\sigma_{1}} \cdots g_{\sigma_{2 k}}$ and hence $1=g_{\sigma} g_{\sigma_{1}} \cdots g_{\sigma_{2 k}}$, so for every $\xi$ we get

$$
n_{\xi}=(-1)^{2 k+1} n_{\xi g_{\sigma} g_{\sigma_{1}} \cdots g_{\sigma_{2 k}}}=-n_{\xi}
$$

which implies that $n_{\xi}=0$ and hence ker $\partial=0$ and so $Y^{\circ}$ is non-orientable.

We restate the orientability criteria of Theorem 3.1 for real toric varieties.

Theorem 3.2 Let $Y$ be a real toric variety defined by a fan $\Sigma$. Then $Y^{\circ}$ is orientable if and only if there exists a basis of $\{ \pm 1\}^{n}$ such that $(-1)^{b}$ is a product of an odd number of basis vectors, for each primitive vector b lying on a ray of $\Sigma$.

The condition of Theorem 3.2 is easily checked. 
Lemma 3.3 Given $A \subset\{ \pm 1\}^{n}$, the condition that there exists a basis of $\{ \pm 1\}^{n}$ such that each vector in A is a product of an odd number of basis vectors, is equivalent to the condition that no product of an odd number of vectors in $A$ is equal to 1 in $\{ \pm 1\}^{n}$.

Proof If we had $v_{1} \cdots v_{2 k+1}=1$, then $v_{2 k+1}=v_{1} \cdots v_{2 k}$, and expressing each $v_{i}$ as the product of an odd number of basis elements of $\{ \pm 1\}^{n}$ yields a contradiction. For the other implication, reduce $A$ to a linearly independent set $A^{\prime}$ and then extend $A^{\prime}$ to a basis of $\{ \pm 1\}^{n}$. If there were a vector in $A \backslash A^{\prime}$ which is a product of an even number of vectors $v=v_{1} \cdots v_{2 k}$, we would have then had $v \cdot v_{1} \cdots v_{2 k}=1$.

We may check if the condition is satisfied by reducing $A$ to a linearly independent set $A^{\prime}$ and checking if each vector in $A \backslash A^{\prime}$ is a product of an odd number vectors in $A^{\prime}$.

The analog of Theorem 3.1 for spherical toric varieties has a similar proof.

Theorem 3.4 Let $Y_{\Delta}^{+} \subset \mathbb{S}^{\Delta}$ be a spherical toric variety defined by a full-dimensional lattice polytope $\Delta \subset \mathbb{R}^{n}$.

(1) $Y_{\Delta}^{+}$is orientable if and only if there exists a basis of $\{ \pm 1\}^{n+1}$ such that for each facet $F$ of $\Delta$ with primitive normal vector $b$, the element $(-1)^{(b, b \cdot F)}$ is a product of an odd number of basis elements.

(2) The components of $Y_{\Delta}^{+}$are naturally indexed by

$$
\left.\{ \pm 1\}^{n+1} /\left\langle(-1)^{(b, b \cdot F)}\right| b \text { is a primitive normal vector to a facet } F \text { of } \Delta\right\rangle \text {. }
$$

\section{Examples and Applications to Lower Bounds}

We settle questions of orientability left open in [20] and explain our motivation from the study of real solutions to systems of polynomials. We begin with an example.

\subsection{Cross Polytopes}

The cross polytope is the convex hull of the basis vectors $e_{1}, \ldots, e_{n}$ in $\mathbb{R}^{n}$ and their negatives $-e_{1}, \ldots,-e_{n}$. When $n>1$ the corresponding toric variety is singular. The rays of its normal fan have generators $( \pm 1, \ldots, \pm 1)$, all with the same image in $(\mathbb{Z} / 2 \mathbb{Z})^{n}$. The hypotheses of Theorem 3.1 hold, and so the corresponding real toric variety is orientable and its smooth points have $2^{n-1}$ connected components. Figure 1 displays the cross polytope when $n=2$ and an embedding in $\mathbb{R}^{3}$ of the corresponding real toric variety.

This example was treated in detail in [21, § 7].

\subsection{Order Polytopes}

The order polytope $O(P)$ [22] of a finite poset $P$ is

$$
O(P):=\left\{y \in[0,1]^{P} \mid a \leq b \text { in } P \Rightarrow y_{a} \leq y_{b}\right\} .
$$



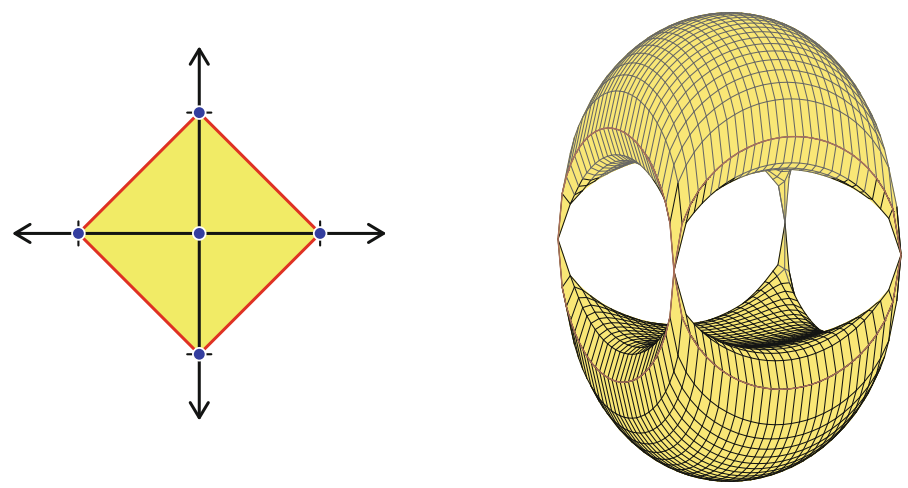

Fig. 1 Two-dimensional cross polytope and double pillow

The integer points of $O(P)$ are its vertices and they correspond to the order ideals of $P$.

Theorem 4.1 $Y_{O(P)}$ is orientable if and only if all maximal chains of $P$ have odd length.

Proof Lemma 4.9 of [20] (or rather its proof) implies that $Y_{O(P)}$ is orientable if all maximal chains of $P$ have odd length. We establish the converse.

The order polytope has three types of facets

$$
\begin{array}{ll}
y_{a}=0 & \text { for } a \in P \text { minimal, } \\
y_{b}=1 & \text { for } b \in P \text { maximal, } \\
y_{b}-y_{a}=0 & \text { for } b \text { covering } a(a \lessdot b) \text { in } P .
\end{array}
$$

Replacing $=$ by $\geq$ gives valid inequalities for $O(P)$, which we write in matrix form

$$
O(P):=\left\{y \in \mathbb{R}^{P} \mid \mathcal{A} y \geq c\right\} .
$$

By Theorem 3.2, $Y_{O(P)}$ is orientable if and only if there is a basis of the row space of $\mathcal{A}$, reduced modulo 2 , such that each row is a sum of an odd number of basis vectors.

Fix a maximal chain $a_{1} \lessdot \cdots \lessdot a_{k}$ in $P$. The corresponding facets of $O(P)$ are

$$
y_{a_{1}}=0, \quad y_{a_{2}}-y_{a_{1}}=0, \quad \ldots, \quad y_{a_{k}}-y_{a_{k-1}}=0, \quad y_{a_{k}}=1,
$$

and the corresponding rows of the matrix $\mathcal{A}$ (modulo 2) are

$$
\left[\begin{array}{ccccccccc}
1 & 0 & 0 & \ldots & 0 & 0 & 0 & \ldots & 0 \\
1 & 1 & 0 & \ldots & 0 & 0 & 0 & \ldots & 0 \\
0 & 1 & 1 & \ldots & 0 & 0 & 0 & \ldots & 0 \\
\vdots & \vdots & \vdots & \ddots & \vdots & \vdots & \vdots & \ldots & \vdots \\
0 & 0 & 0 & \ldots & 1 & 0 & 0 & \ldots & 0 \\
0 & 0 & 0 & \ldots & 1 & 1 & 0 & \ldots & 0 \\
0 & 0 & 0 & \ldots & 0 & 1 & 0 & \ldots & 0
\end{array}\right]
$$


where the non-zero columns correespond to $a_{1}, \ldots, a_{k}$. This gives $k+1$ rows whose sum is zero modulo 2. If $k$ is even, Lemma 3.3 implies that $Y_{O(P)}$ is non-orientable.

A poset $P$ is ranked modulo 2 if all maximal chains in $P$ have the same parity.

Theorem 4.2 A spherical toric variety $Y_{O(P)}^{+}$is orientable if and only if $P$ is ranked modulo 2.

Proof By Lemma 4.9 of [20], $Y_{O(P)}^{+}$is orientable if it is ranked modulo 2.

Suppose that $P$ is not ranked modulo 2. We exhibit an odd number of rows of the augmented matrix $[\mathcal{A}: c]$ whose sum is zero modulo 2, which shows that $Y_{O(P)}^{+}$is not orientable, by Theorem 3.4 and Lemma 3.3, as these rows have the form $(b, b \cdot F)$ for $b$ a primitive normal to a facet of the order polytope.

The order polytope is defined by the facet inequalities (4.1). For a maximal chain $a_{1} \lessdot \cdots \lessdot a_{k}$ in $P$, the corresponding rows of the augmented matrix $[\mathcal{A}: c]$ are

$$
\left[\begin{array}{ccccccccc|c}
1 & 0 & 0 & \ldots & 0 & 0 & 0 & \ldots & 0 & 0 \\
1 & 1 & 0 & \ldots & 0 & 0 & 0 & \ldots & 0 & 0 \\
0 & 1 & 1 & \ldots & 0 & 0 & 0 & \ldots & 0 & 0 \\
\vdots & \vdots & \vdots & \ddots & \vdots & \vdots & \vdots & \ldots & \vdots & \vdots \\
0 & 0 & 0 & \ldots & 1 & 0 & 0 & \ldots & 0 & 0 \\
0 & 0 & 0 & \ldots & 1 & 1 & 0 & \ldots & 0 & 0 \\
0 & 0 & 0 & \ldots & 0 & 1 & 0 & \ldots & 0 & 1
\end{array}\right]
$$

Observe that the sum of these rows is $[0: 1]$. Each row of $[\mathcal{A}: c]$ has the form $(b, b \cdot F)$ (modulo 2), where $b$ is a primitive normal vector to a facet $F$ of $\Delta$.

Since $P$ is not ranked modulo 2 , it has two maximal chains of different parities. Summing the rows of $[\mathcal{A}: c]$ which correspond to facets given by the two chains gives a sum of an odd number of rows of $[\mathcal{A}: c]$ which is equal to zero modulo 2 .

\subsection{Real Solutions to Systems of Equations}

In [20] we considered systems,

$$
f_{1}\left(x_{1}, \ldots, x_{n}\right)=f_{2}\left(x_{1}, \ldots, x_{n}\right)=\cdots=f_{n}\left(x_{1}, \ldots, x_{n}\right)=0
$$

where each $f_{i}$ is a real polynomial whose exponent vectors lie in $\Delta \cap \mathbb{Z}^{n}$, for a fixed lattice polytope $\Delta$, called the Newton polytope of the system. When the exponent vectors $\Delta \cap \mathbb{Z}^{n}$ affinely span $\mathbb{Z}^{n}$, the solutions to (4.2) correspond to a linear section $L \cap Y_{\Delta}$ of the real projective toric variety $Y_{\Delta}$ corresponding to $\Delta$. Here $L \subset \mathbb{R P}^{\Delta}$ is a linear subspace of codimension $n$. Projecting from a general codimension one linear subspace $E$ of $L$, we may realize the solutions to (4.2) as the fibers of a map

$$
\pi_{E}: Y_{\Delta} \longrightarrow \mathbb{R P}^{n}
$$

to real projective space. If $n$ is odd, then $\mathbb{R P}^{n}$ is orientable. If $Y_{\Delta}$ is also orientable, then fixing orientations, the map $\pi_{E}$ has a degree whose absolute value gives a lower 
bound on the cardinality of a fiber of $\pi_{E}$, and thus on the number of real solutions to (4.2).

More generally, we may lift this projection to the spherical toric varieties

$$
\pi_{E}^{+}: Y_{\Delta}^{+} \longrightarrow \mathbb{S}^{n}
$$

If $Y_{\Delta}^{+}$is orientable, we fix an orientation and the absolute value of the degree of $\pi_{E}^{+}$is a lower bound on the number of solutions to the system (4.2). Changing orientations in each component if necessary, we may assume that the degree is divisible by the number of components of $\left(Y_{\Delta}^{+}\right)^{\circ}$.

This has the following consequence for lower bounds to systems of polynomial equations.

Theorem 4.3 Suppose that we have a system of polynomials (4.2) with Newton polytope $\Delta$ where $\Delta \cap \mathbb{Z}^{n}$ affinely spans $\mathbb{Z}^{n}$ whose solutions are a fiber of a projection map $\pi_{E}^{+}$(4.3). If there is a basis for $\{ \pm 1\}^{n+1}$ such that $(-1)^{(b, b \cdot F)}$ is a product of an odd number of basis elements for every primitive normal vector $b$ to a facet $F$ of $\Delta$, then the absolute value of the degree of the map $\pi_{E}^{+}$is a lower bound for the number of real solutions to (4.2), and this lower bound is a multiple of the number of components of $\left(Y_{\Delta}^{+}\right)^{\circ}$.

Moreover, the map $\pi_{E}^{+}$does not have a degree if this condition is not satisfied.

Remark 4.4 We did not need to consider the parity of $n$, for the condition of Theorem 3.2 implies that of Theorem 3.4. (A vector lies in a ray of the normal fan $\Sigma$ to $\Delta$ if and only if it is normal to a facet $F$ of $\Delta$.)

\subsection{Conclusions}

We characterized the orientability of $Y_{\Delta}$ and $Y_{\Delta}^{+}$, which implies that the corresponding polynomial system has lower bounds on its number of real solutions, expressed as the degree of a projection $\pi_{E}$ or $\pi_{E}^{+}$. These degrees have been computed for polynomial systems from posets [20] and those from foldable triangulations [12,13,20]. Our characterization of orientability replaces the condition in [20] that a variety is Cox-oriented and therefore strengthens the results of [20], particularly Theorem 3.5.

Acknowledgments The research of Sottile was supported in part by NSF grant DMS-1001615, and the Mittag-Leffler Institut.

\section{References}

1. Azar, M., Gabrielov, A.: Lower bounds in B. and M. Shapiro conjecture. Discrete Comput. Geom. 46(4), 636-659 (2011)

2. Craciun, G., Dickenstein, A., Shiu, A., Sturmfels, B.: Toric dynamical systems. J. Symb. Comput. 44(11), 1551-1565 (2009)

3. Davis, M.W., Januszkiewicz, T.: Convex polytopes, Coxeter orbifolds and torus actions. Duke Math. J. 62(2), 417-451 (1991)

4. Degtyarev, A.I., Kharlamov, V.M.: Topological properties of real algebraic varieties: Rokhlin's way. Uspekhi Mat. Nauk 55(4(334)), 129-212 (2000) 
5. Delaunay, C.: Real structures on compact toric varieties. Ph.D. thesis, Université Louis Pasteur, Strasbourg (2004)

6. Eremenko, A., Gabrielov, A.: Degrees of real Wronski maps. Discrete Comput. Geom. 28(3), 331-347 (2002)

7. Finashin, F., Kharlamov, V.: Abundance of real lines on real projective hypersurfaces. Int. Math. Res. Notices (2012). doi:10.1093/imrn/rns135

8. Fulton, W.: Introduction to Toric Varieties. Annals of Mathematics Studies, vol. 131. Princeton University Press, Princeton (1993)

9. García-Puente, L., Hein, N., Hillar, C., Martín del Campo, A., Ruffo, J., Sottile, F., Teitler, Z.: The secant conjecture in the real Schubert calculus. Exp. Math. 21(3), 252-265 (2012)

10. Hein, N., Sottile, F.: Lower bounds in real Schubert calculus. http://arXiv.org/1211.7160 (2013)

11. Hein, N., Sottile, F., Zelenko, I.: A congruence modulo four in real Schubert calculus. http://arXiv/ abs/1211.7160 (2012)

12. Joswig, M., Witte, N.: Products of foldable triangulations. Adv. Math. 210(2), 769-796 (2007)

13. Joswig, M., Ziegler, G.: Foldable triangulations of lattice polygons. Am. Math. Mon. to appear. http:// arXiv/abs/1207.6865 (2012)

14. Krasauskas, R.: Toric surface patches: advances in geometrical algorithms and representations. Adv. Comput. Math. 17(1-2), 89-113 (2002)

15. Nakayama, H., Nishimura, Y.: The orientability of small covers and coloring simple polytopes. Osaka J. Math. 42(1), 243-256 (2005)

16. Okonek, Ch., Teleman, A.: Intrinsic signs and lower bounds in real algebraic geometry. J. Reine Angew. Math. to appear. http://arXiv/abs/1112.3851 (2012)

17. Okonek, Ch., Teleman, A.: A wall crossing formula for degrees of real central projections. http://arXiv/ abs/1206.4271 (2012)

18. Pachter, L., Sturmfels, B. (eds.): Algebraic Statistics for Computational Biology. Cambridge University Press, New York (2005)

19. Ruffo, J., Sivan, Y., Soprunova, E., Sottile, F.: Experimentation and conjectures in the real Schubert calculus for flag manifolds. Exp. Math. 15(2), 199-221 (2006)

20. Soprunova, E., Sottile, F.: Lower bounds for real solutions to sparse polynomial systems. Adv. Math. 204(1), 116-151 (2006)

21. Sottile, F.: Toric ideals, real toric varieties, and the moment map. In: Topics in Algebraic Geometry and Geometric Modeling. Contemporary Mathematics, vol. 334. American Mathematical Society, Providence, RI, pp. 225-240 (2003)

22. Stanley, R.P.: Two poset polytopes. Discrete Comput. Geom. 1(1), 9-23 (1986)

23. Welschinger, J.-Y.: Invariants of real rational symplectic 4-manifolds and lower bounds in real enumerative geometry. C. R. Math. Acad. Sci. Paris 336(4), 341-344 (2003) 